\title{
Development of Personalized Flexible Report for Manufacturing Execution System Based on Data Warehouse Technology
}

\author{
Hsiu-Li Liao ${ }^{1 *}$, Chen-Huei Chou ${ }^{2}$, Kuo-Chen Li $^{1}$, Yi-Chun Lin ${ }^{1}$ \\ ${ }^{1}$ Information Management Department Chung Yuan Christian University, Taoyuan City, Taiwan, R. O. C. \\ 2 Dept. of Supply Chain and Information Management, College of Charleston, Charleston, SC, USA. \\ * Corresponding author. Tel.: 18439532278; email: chouc@cofc.edu \\ Manuscript submitted June 2, 2016; accepted August 15, 2016.
}

doi: 10.17706/ijcee.2016.8.4.280-287

\begin{abstract}
In an attempt to effectively manage the collection of information within their operations, manufacturing enterprises have implemented Manufacturing Execution Systems. One of the notable disadvantages of the traditional report system is its lack of a flexible query mechanism, which leads to poor query performance. This research attempts to develop a prototype of a personalized flexible report system in which users can themselves set query information to support existing business intelligence systems or a traditional report system. Based on the interviews of actual users, we developed a personalized flexible report prototype system to satisfy their needs. This improvement in the report system reduces the work load of information personnel and, thereby, improves work efficiency.
\end{abstract}

Key words: Manufacturing execution system, personalized flexible report, data warehouse, IC packaging and testing industry.

\section{Introduction}

In an attempt to effectively manage the collection of information within their operations, manufacturing enterprises have implemented Manufacturing Execution Systems (MES). The Advanced Manufacturing Research (AMR) organization defines MES as "an integrated architecture for plantwide information management that groups applications and functions around a central common database used to share product and process data among the applications" [1], [2]. The Manufacturing Enterprise Solutions Association (MESA) indicates that "MES delivers information that enables the optimization of production activities from order launch to finished goods. Using current and accurate data, MES guides, initiates, responds to, and reports on plant activities as they occur" [3].

One of the notable disadvantages of the traditional report system is its lack of a flexible query mechanism, which leads to poor query performance. Due to different report requirements among users playing different roles, a huge amount of complex-and mostly unnecessary-data is displayed in each user's report. Business intelligence (BI) systems are "specialized tools for data analysis, query and reporting, that support organizational decision-making that potentially enhances the performance of a range of business processes" [4]. BI systems use Extract-Transform-Load tools to help discover and correct data quality issues and efficiently load large volumes of data into the warehouse [5] and then present information through BI reports. In order to increase sales and market share, BI system vendors expound a variety of functions, 
which were designed to meet a variety of business needs. Therefore, as business needs expand, functions of BI systems become increasingly complex and-as a result-increasingly difficult for users to learn.

In order to overcome the issues of complex standard report, this research attempts to develop a prototype of a personalized flexible report system in which users can themselves set query information to support existing BI systems or a traditional report system. The purposes of the study are as follows: (1) to understand the structural features of Data Warehouse and Manufacturing Execution Systems; (2) to learn the data features of MES in the packaging and testing industry; (3) to provide a way for users to get personalized reports quickly based on personal requirements.

\section{Research Method and System Structure}

We conducted semi-structured interviews for requirement analysis for a personalized flexible report system. AA company was chosen for the development of the prototyping report system. AA company is among the top five IC packaging and testing manufacturers in the world. Its largest manufacturing plants are located in South Korea, Shanghai, the Philippines and Taiwan with 2,600 employees in Taiwan alone. The main packaging and testing technologies used by AA company are Bumping, Circuit Probing, Flip Chip, Final Test and Lead Frame. AA company has six production lines, including Lead Frame, Circuit Probing, Die Package Service, Flip Chip, Final Test and Wafer Bumping.

\subsection{Requirement Analysis}

The case company generates manufacturing reports for production line department, production management department, and quality management department. Workers can be further classified into operator, engineer, manager, order entry personnel, planner. Different workers in different department have different needs for report. For example, engineers in production line department need yield, output, scrap, transaction history, while engineers in quality management department need group hold, future hold and scrap report. A traditional unified reporting system showing everything for every user may not work in the case company.

We used semi-structured method to interview eight experienced workers with more than five-year experience from different departments. The results of the interviews showed that the reporting system of MES should:

- provide correct production information, speed up data query, provide flexible operation interface and improving flexible settings and management;

- simplify the query operation, increase user convenience and help users to reduce the waiting time for generating a report;

- provide users with the information after a comprehensive analysis and allow flexible customization so that users can query the information they need more efficiently.

\subsection{System Structure}

Based on the suggestions found in requirement analysis, we proposed a system structure to include user authority, data query setting, and personalized report (see Figure 1) for the development of the personalized flexible report system.

User authority module help system administrator set system functions for users to operate based on users' group and authority. Data query setting module let system administrator set table, data field and data relation to show the information user's query based on users' group and authority. The personalized report module allows users to set personal query criteria and field by themselves and then store the settings after they login to the report system. 

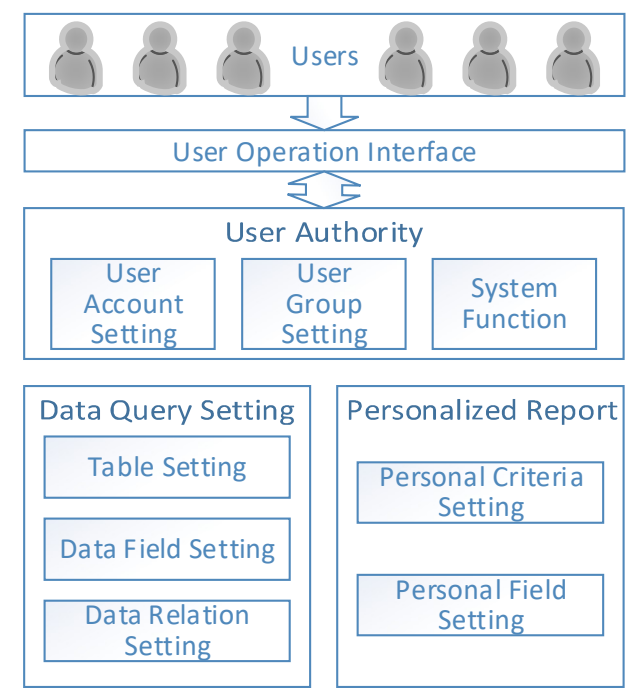

Fig. 1. System Structure of Personalized Flexible Report.

\subsection{System Structure}

We followed a three-tier architecture (see Fig. 2) to develop the personalized report system. All data is stored in a Data Warehouse server. Client users used browser to interact with Application Server to generate their own personalized report. Users do not need powerful computing devices to complete their tasks since the major computing is on the server side.

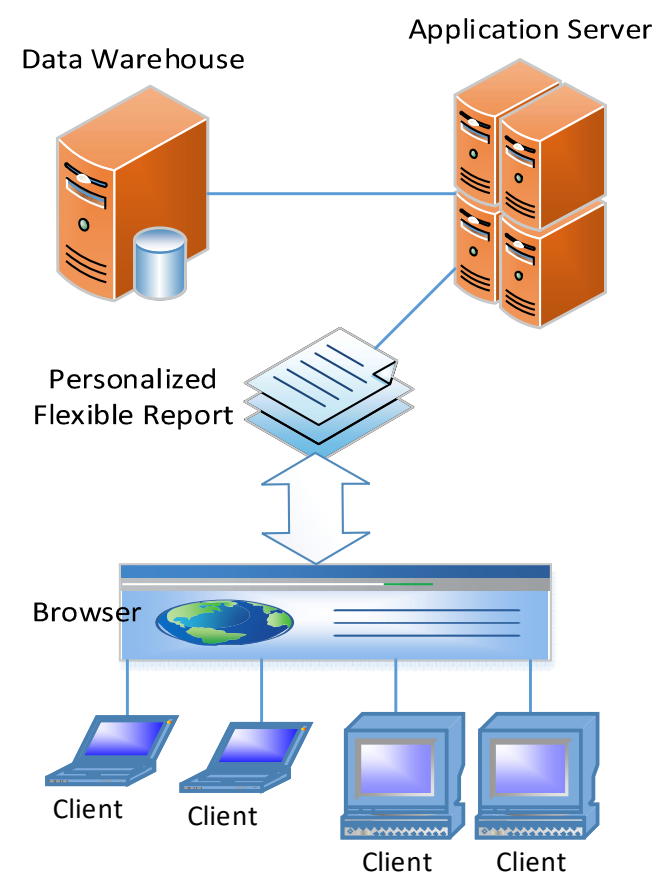

Fig. 2. Information Architecture of Personalized Flexible Report.

\section{Prototype Development}

We followed the relational database approach [6] to develop the personalized flexible report system. 11 tables listed in Fig. 3 were created to satisfy the system requirements. The purpose of each table is listed below:

- PR_User: to store user's account, password and group 
- PR_Group: to store each group name

- PR_Function: to store each system function, functions type and link address

- PR_FunctionType: to store each function type

- PR_GroupAuthority: to store usable functions of each group

- PR_Table: to store system table users can query

- PR_TableType: to store each type of system table users query

- PR_TableDetail: to store the detailed fields of system table

- PR_TableJoin: to store the relational fields of system table

- PR_GroupTableType: to store table type each group can query

- PR_Condition: to store each user's personal report criteria

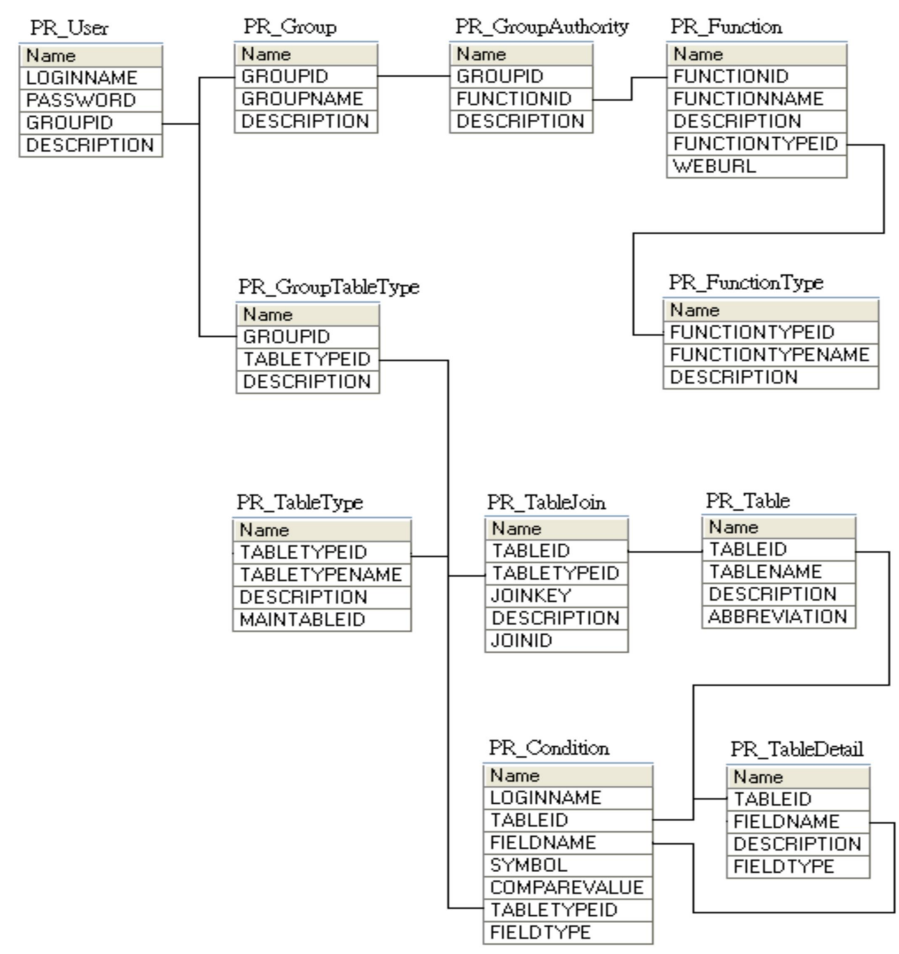

Fig. 3. Relationships in the Database Development.

Next, we discussed the details of three modules in the system.

\subsection{Abbreviations and Acronyms}

The main purpose of this module is to use user group to simplify the process by assigning functions available to users based on their roles. The code below shows the way to join the corresponding table.

Select u.loginname,g.groupname,f.functionname,ft.functiontypename,f.weburl ,ft.functiontypeid,f.functionid

from PR_user u

join PR_group g on u.groupid=g.groupid

join PR_groupauthority ga on g.groupid=ga.groupid

join PR_function $f$ on ga.functionid=f.functionid

join PR_functiontype $\mathrm{ft}$ on f.functiontypeid=ft.functiontypeid

where u.loginname='admin'

The entity relationship diagram in Figure 4 shows the details of this module. 


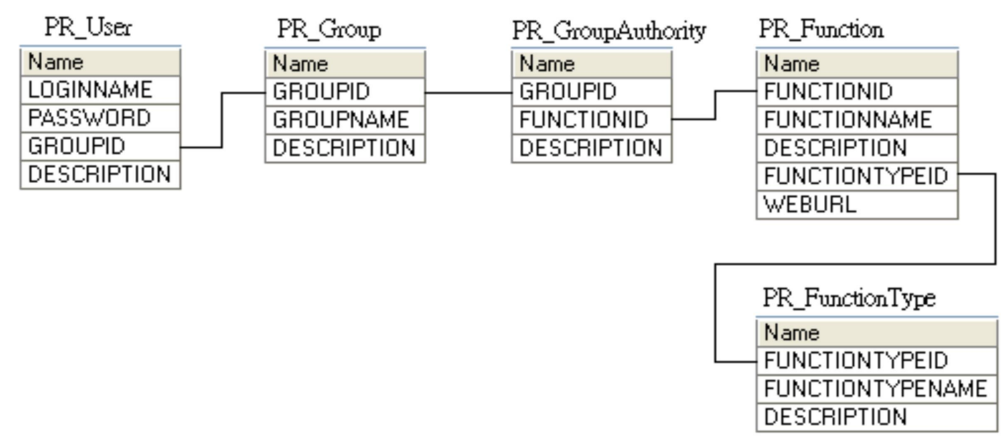

Fig. 4. Relationships for User Authority Module.

\subsection{Data Query Setting}

Based on the user group of a user, this module help link all required tables together in order to build the overall view of data inquiry based on users' requests. The code below shows the preparation and Figure 5 shows the relationships.
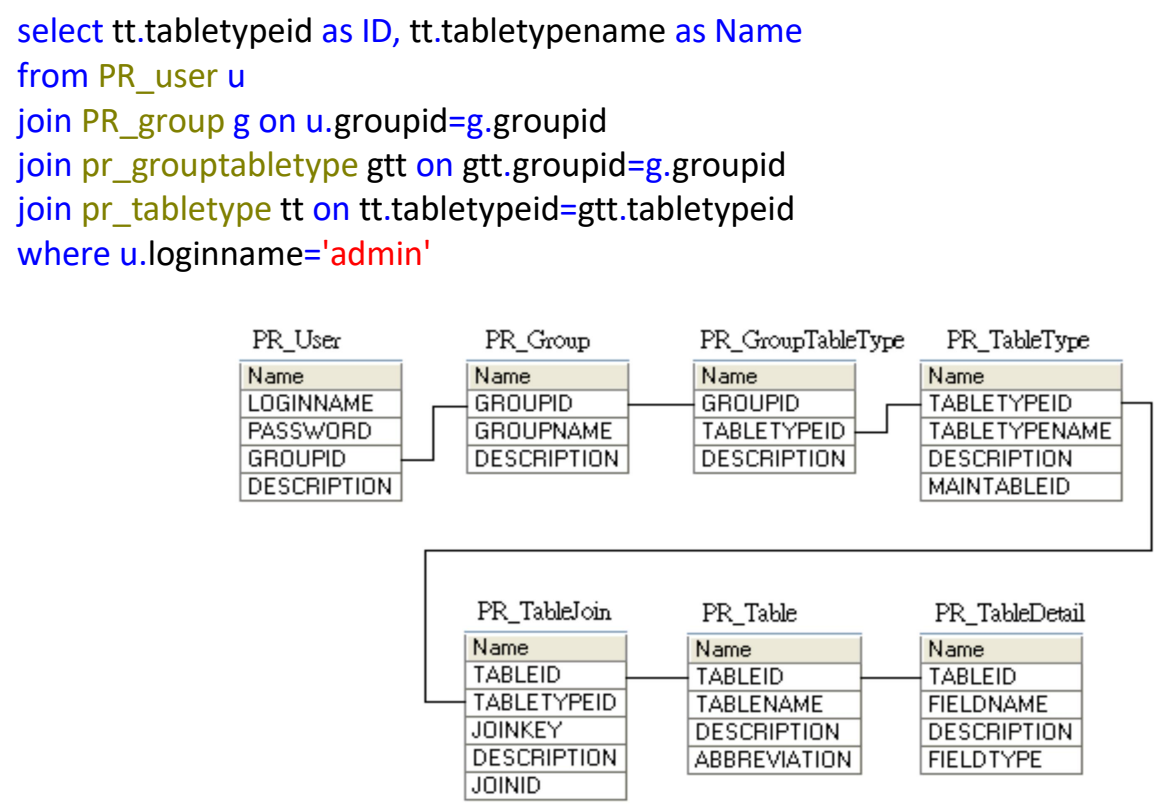

Fig. 5. Relationships for Data Query Setting Module.

\subsection{Other Recommendations}

Users may need to generate different reports from time to time. They may need to generate the same report style later. This module helps user to save personal setting according to the criteria and details of queries. When the user needs to perform the same query again, he or she loads the pre-stored settings to save time. The code used to join the tables is listed below and the relationships diagram is listed in Figure 6.

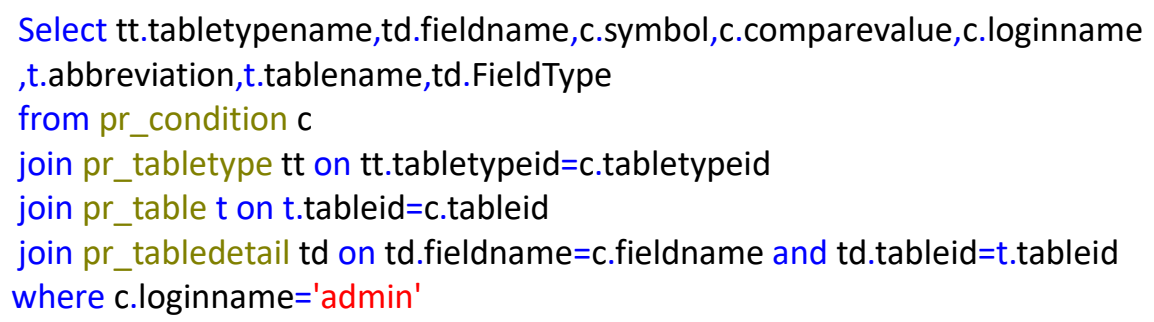




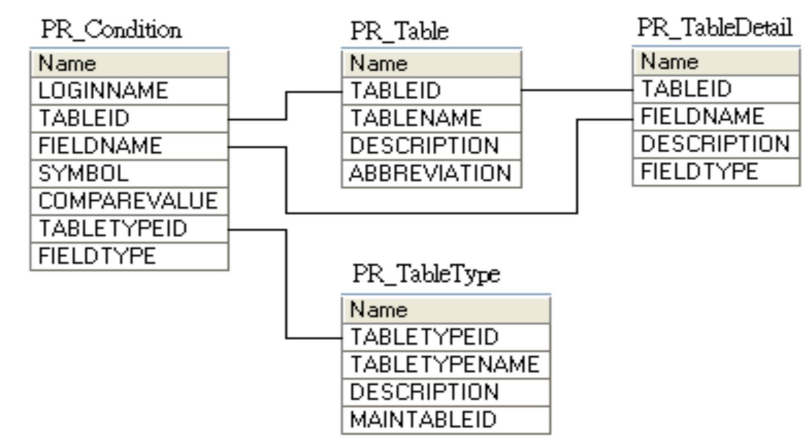

Fig. 6. Relationships for Data Query Setting Module.

\subsection{Other Recommendations}

Oracle relational database was used for data warehouse and the adjusted data were used to speed up the query report. Data warehouse of the report system was built on Sun Solaris operating system. The application server was developed using Microsoft Visual Studio .Net 2003-Asp.Net. The application server ran on Microsoft Windows Server 2003 with the Internet Information Service hosted to take browsers' request from the clients.

\section{System Validation}

We tested our prototype using the workers in the interview process. They were experienced workers with more than five years of experience. They also worked from different departments with different roles. The coverage of user role for the validation was satisfied. Based on the features they need to use for their jobs, they used the prototype to apply queries, store personal settings, and reuse the stored criteria and settings. Requirements were met and they found it beneficial. Flexibility and efficiency were reflected in the validation process. None issues were reported and flexible query mechanism was ensured.

\section{Discussion and Conclusion}

One disadvantage of the traditional report system is its lack of a flexible query mechanism. This shortcoming leads to poor query performance, namely cumbersome reports that take a long time to produce. Because the requirements for users vary considerably, the reports become vast and complex. From the perspective of information management personnel, requirements to increase and decrease report fields are numerous. Too much time is needed to maintain users' reports. Although a BI system provides a flexible query service, many functions are not changed according to the user's requirements. Additionally, system vendors focus on varied, powerful functions to increase their sales and market share. In reality, the system functions are complex and difficult to learn and use effectively.

After interviewing several production managers, researchers in this study found that the personalized flexible report system with simple settings and operation interface can help users receive queried information faster as well as help production management personnel find problems. Although the personalized flexible report system cannot support requirements for customization that are too specific, it reduces the amount of similar and repetitive content that would otherwise have been included. Also, it helps reduce the proposed change requirements. Information personnel consider that reducing the revision times of one report can decrease the time spent revising or developing other, newer reports. This improvement in the report system reduces the work load of information personnel and, thereby, improves work efficiency.

However, this work utilizes a specific industry. If functions are set and changed accordingly based on a 
new application, generalizability might not be an issue. Future works may extend this study to different applications and industries.

\section{References}

[1] Samanich, N. J. (1993). Understand your requirements before choosing an MES. Manufacturing Systems, 34-39.

[2] Cheng, F. T., Chen, E., Deng, J. Y., \& Nguyen, K. (1999). Development of a system framework for the computer-integrated manufacturing execution system: A distributed object-oriented approach. International Journal of Computer Integrated Manufacturing, 5, 384-402.

[3] MESA International (1997). MES explained: a high level vision, 6, 9.

[4] Elbashir, M. Z., Collier, P. A., \& Davern, M. J. (2008). Measuring the effects of business intelligence systems: the relationship between business process and organizational performance. International Journal of Accounting Information Systems, 3, 135-153.

[5] Chaudhuri, S., Dayal U., \& Narasayya V. (2011). An overview of business intelligence technology. Communications of the ACM, 8, 88-98.

[6] Chen, P. P. S. (1976). The entity-relationship model-toward a unified view of data. ACM Transactions on Database Systems (TODS), 1(1), 9-36.

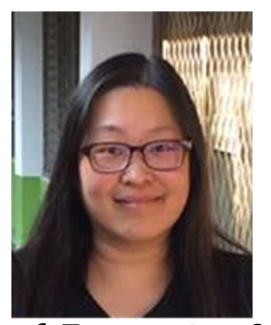

Hsiu-Li Liao received her Ph.D. in information management from National Taiwan University Science and Technology in 2008. She is currently working as an Associate Professor at the Department of Information Management at Chung Yuan Christian University. She has published refereed papers in Computers \& Education, Computers in Human Behavior, Eurasia Journal of Mathematics, Science and Technology Education, Internet Research, International Journal of Services Technology and Management, Review of Economics \& Finance, Social Behavior and Personality, International Journal of Electronic Business Management, Lecture Notes in Computer Science, and other Chinese management journals. She is also a reviewer of eight IS international journals. She is a member of IACIS and SIim.

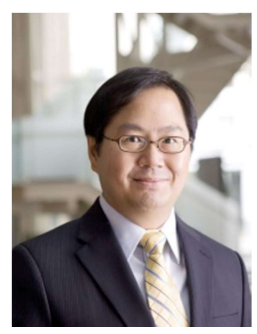

Chen-Huei Chou is an Associate Professor of Information Management and Decision Sciences in the School of Business at the College of Charleston, SC, U.S.A. His areas of interests include web design issues in disaster management, ontology development, Internet abuse in the workplace, text mining, data mining, and knowledge management. His research has been published in MIS journals and major conference proceedings, including MIS Quarterly, Journal of Association for Information Systems, Decision Support Systems, IEEE Transactions on Systems, Man, and Cybernetics, Computers in Human Behavior, Internet Research, and Journal of Information Systems and e-Business Management.

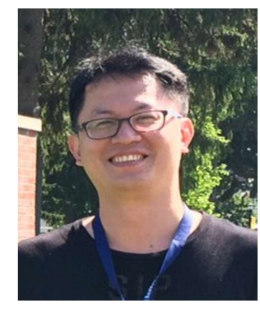

Kuo-Chen Li received his Ph.D. degree of Computer Science and Engineering from University of Louisville in 2008. He was a postdoctoral fellow in Information and Computer Engineering department at Chung Yuan Christian University between 2009 and 2010. He is currently an assistant professor of Information Management department at Chung Yuan Christian University from 2011. His research topics include Digital Learning, Mobile Commerce, Artificial Intelligence, Neural Networks, and Data mining. 
Yi-Chun Lin received her Master degree in Information Management from Chung Yuan Christian University in 2011. Her research interests include manufacturing execution system, data warehouse, business intelligence, IC packaging and testing industry, and management information system. 